\title{
The Effects of Valence and Arousal on Time Perception in Depressed Patients
}

\section{Jeong-Won Choi \\ Gi-Eun Lee \\ Jang-Han Lee}

Department of Psychology, Chung-Ang University, Seoul, Korea

\author{
This article was published in the following Dove Press journal: \\ Psychology Research and Behavior Management
}

\begin{abstract}
Purpose: The aim of the present study was to examine the effects of emotional state on time perception in patients with depression and to investigate if time perception in patients with depression could be changed by induced emotional state.
\end{abstract}

Patients and Methods: A clinically-depressed (CD, n=19) and non-depressed (ND, n=22) group viewed four video clips of different levels of valence and arousal (ie, positive-high arousal, positive-low arousal, negative-high arousal, negative-low arousal). After inducing emotion state, all participants performed a time perception task to measure differences in the perception of time. Results: The results showed that the CD group perceived time passing more slowly than the ND group at baseline and in all conditions, especially in the negative-low arousal condition. The ND group perceived time passed more quickly in the positive-high arousal condition compared to the $\mathrm{CD}$ group. These results indicate that emotional state with combined valence and arousal factors could change time perception in patients with depression.

Conclusion: Based on these results, it is suggested that changing the emotional state of the depressive patient by considering valence as well as arousal is important to improve the distortion on time perception.

Keywords: depression, time perception, valence, arousal, internal clock model

\section{Introduction}

While physical time always flows at a constant speed, psychologically conscious time is estimated as variable and subjective by one's psychological state. If people perceive the passage of time differently according to their emotional state, people with mood disorders will show a difference in time perception from the normal group. ${ }^{1,2}$ As many previous studies revealed, individuals with depression experience the time flow as slower than healthy individuals and have difficulty in estimating how much time has passed. ${ }^{3-6}$ Therefore, individuals with pathological depression need to be included in time perception studies because patients with depression experience lower arousal responses, heightened negative emotions, and attenuated positive emotions compared to the individuals without depression. ${ }^{7}$

Although no specific model has been proposed to explain the time perception of individuals with depression, the internal clock model which explains time perception in normal individuals may be extended to address the time perception of individuals with depression. ${ }^{8}$ The internal clock model postulates that the processing of time perception depends on internal factors of organisms. Specifically, individuals have an internal clock system as pacemaker, accumulator, and pulses, which is the smallest unit of time. According to this model, the main operating
Correspondence: Jang-Han Lee

Department of Psychology, Chung-Ang

University, Seoul 06974, Korea

Tel +8228205751

Fax +8228165124

Email clipsy@cau.ac.kr 
mechanisms of the internal clock system are physiological responses such as arousal. The structure of the pacemaker produces physiological responses, which indicate units of internal temporal information (arousal) in a regular rate. Induced arousal responses pass through a gate to an accumulator; in the accumulator, temporal units accumulate and are conveyed to the memory system. Two memory systems (working memory and reference memory) compare the arousal responses to a previously existing arousal rate. Through this processing, a comparator estimates the flow of time. ${ }^{9,10}$ The internal clock speeds up with increasing amounts of arousal and more pulses are emitted, which results in the perception of time passing more slowly. ${ }^{8,11,12}$ Recently, several studies have revealed that emotion modulates time perception by arousal factor. ${ }^{13,14}$ Furthermore, according to this internal clock model, there are some evidences that individuals with a depressive trait or a patient with depression has a slower perception of time. ${ }^{5,15}$ Considering the effect of arousal on the internal clock, the arousal responses and imbalanced emotion led to different patterns of time perception in individuals with and without depression depending on the emotional context.

Although the internal clock model provided a perspective for explaining human time perceptionrelated behavior according to arousal level, the explanation for cognitive factors related to human attention allocation is somewhat insufficient. An attention-gate model is the model which can compensate for these limitations. According to Zakay and Block, ${ }^{16}$ human's perception of time is controlled by the attention allocation which regulates the flow of pulses in the accumulator by the switch. When the timing of an event begins, attention resources are allocated to the switch and as the switch closes, pulses generate by the pacemaker begin to accumulate in the accumulator. In contrast, when the switch is open, pulses are blocked. Through this process, the accumulator calculates the passage of time by summing the number of pulses input during a predetermined time interval. At this time, if more pulses are accumulated, it is estimated that the time interval is longer.

In terms of these mechanisms, both arousal level and attention resources allocation can lead to changes in time perception. Perhaps a high level of arousal and the allocation of attention resources will affect an individual's lengthening time perception. Hence, it is explained that those who report depressive mood tend to perceive time more slowly than no-depressive individual as a result of the faster running internal clock. The high level of arousal is often shown when fronting the threatening stimuli and humans are usually shown to allocate more attention to negative stimuli than positive ones. The degree of perception of external information also has a subjective characteristic like time perception, emotionally anxious or depressed people tend to perceive external stimuli a little more negatively, so it seems that they will always have a higher level of arousal than the general group. Besides, when individuals with depression experience negative emotions, they often think about the cause and effect of these emotions repeatedly (referred to as rumination). Ruminative thought is more likely to induce depression again, resulting in triggering the vicious cycle of depression. . $^{2,3,17}$

As outlined, emotions are known to play an important role in the perception of the external environment of human-beings; these emotions consist of various and complex factors. According to Russell, ${ }^{18}$ emotion is classified by two axes of arousal (low-to-high arousal) and valence (negative to positive valence). Based on these axes, emotional states can be represented at any level of valence and arousal or a neutral level of one or both of these factors on these four dimensions. Because of this diversity of emotions, the level of perception will be different depending on the combination of arousal or valence, which can affect time perception as well. For example, some of the previous studies found that high-arousal negative stimuli led to the perception of time passing more slowly than higharousal positive stimuli. In contrast, low-arousal positive stimuli led to the perception of time passing more slowly than low-arousal negative stimuli. ${ }^{12,19}$ According to recent studies, it is possible to assume that humans can automatically show attention avoidance to negative stimuli for emotional regulation at high arousal level, so it can be estimated that time perception was less at high arousal levels. $^{20}$

Although various research on the influence of emotion on time perception was carried out, there are many areas that are not yet clear about the effect of emotion on time perception due to the use of unstandardized emotion stimuli or methodological differences in experimental procedures. For example, several studies used the VAS and an estimation task in order to measure time perception. These tasks may be influenced by participants' subjective responses and resulted in measuring perception of time inaccurately. In order to compensate these limitations, recent studies used validated and standardized emotional 
stimuli as International Affective Picture System (IAPS), International Affective Digitalized Sounds System (IADS), and facial expressions. These different standardized emotional stimuli have shown the evidence that the negative emotion and high arousal were judged longer than neutral stimuli. However, most studies are limited to investigate the judgment of the time of emotional stimuli, and do not fully explain the effect of emotional states of individuals on time perception. ${ }^{13,14,19,21}$

In addition, a recent meta-study suggested that it is difficult to find a clear consensus on the relationship between depressive emotions and time perception. In this study, four types of time perception tasks were compared, and there were no significant effects of depression on time perception. According to the results of this study, time perception in depression is in line with the internal clock system. In general, the depressive participants were judging time less quickly with a faster running internal clock, but they were shown to also lack a significant effect of emotion on time perception at some tasks. As a result, they announced that the empirical findings on time perception in depressive participants were not inconsistent and do not fully support a faster running internal clock hypothesis. ${ }^{22}$

However, as mentioned earlier, most of the studies showed the result of reaction to an observed emotional stimulus which interfered with the processing of time and not the result of the effect of an inducing emotional stated on time judgments in a neutral stimulus. For a better interpretation of the relationship between emotion and temporal perception, it is necessary to determine not only time perception of emotional stimuli, but also how neutral external stimuli were perceived depending on the individual's emotional state. To accumulate additional information, the study participants were asked to determine the length of time for neutral stimuli after inducing an emotional state. Furthermore, this study used video clip stimuli to avoid the shortcomings of picture stimuli, which are limited in the induction of the intended valence and arousal responses. $^{23}$ It has been suggested that video clip stimuli overcome the limitation of picture stimuli by providing contextual information. ${ }^{24,25}$

The aim of this study was to examine the effect of emotional state on depressive individuals' subjective time perception of neutral stimuli using a temporal bisection task. This task was based on the inner temporal unit to measure time perception objectively and assumed that the speed of the internal clock of individuals with depression is slower than that of individuals without depression. ${ }^{2}$

\section{Patients and Methods}

\section{Participants}

The participants consisted of two groups: the clinicaldepressive (CD) group and the non-depressive (ND) group. The $\mathrm{CD}$ group was recruited from a community mental health center and the ND group was recruited via a brief description of the experiment in an internet advertisement. For the $\mathrm{CD}$ group, nineteen participants (females=11, Mean age $=35.05, \mathrm{SD}=7.99$ ) were included who had a history of depressive disorders and were having a current depressive episode. The ND group consisted of twenty-two participants (females $=12$, Mean age $=34.45$, $\mathrm{SD}=3.69$ ) who had no history of mental health problems and reported few depressive states. To confirm depressive state prior to the experiment, all candidate participants completed the Beck Depressive Inventory-second edition (BDI-II) and a mental health history. ${ }^{26}$ The level of depression of the $C D$ group was rated severe $(M=34.84$, $\mathrm{SD}=11.95$ ); on the other hand, the ND group exhibited minimal depressive states $(\mathrm{M}=5.77, \mathrm{SD}=3.69)$. According to a brief interview, participants in the $\mathrm{CD}$ group were all diagnosed with major depressive disorder and the mean duration of disorder was 9.7 years (range from 2-27 years). All of the $\mathrm{CD}$ group had at least one type of treatment and six individuals had a history of hospitalization. Fourteen individuals received psychological counseling currently and/or in the past, and nineteen individuals were treating with medication for depression, anxiety, insomnia, or other conditions. Thirteen individuals reported comorbid disorders, including anxiety disorders $(n=13)$, sleep disorders $(n=6)$, eating disorders $(n=2)$, and substance abuse disorders $(n=1)$. Nine individuals had a family history of mental illness in parents $(n=5)$, relatives $(n=3)$, siblings $(n=2)$, or other family members $(n=2$; spouse, child).

\section{Measurements \\ Self-Report Questionnaires}

To measure the level of depression of participants, BDI-II was used. A total score of 13 or less means a low level of depression; on the other hand, a higher total score indicates severe depressive symptoms. Cronbach's $\alpha$ in this study was 0.96 . The two types of anxiety were evaluated by STAI (State-Trait Anxiety Inventory). ${ }^{27}$ STAI-S measures state anxiety about the actual event and STAI-T measures trait anxiety like one's personal characteristic. Total score ranges from $20-80$, with higher 
scores indicating greater anxiety. Cronbach' $\alpha$ was 0.96 for the STAI-S and 0.96 for the STAI-T in this study. To measure both positive and negative affect of participants, PANAS (The Positive Affect and Negative Affect Schedule) was used. ${ }^{28}$ This scale consists of two 10-item subscales: positive affect (PA) and negative affect (NA), rated on a 5-point scale. Cronbach' $\alpha$ in the current study was 0.83 for the PA subscale and 0.96 for the NA subscale.

\section{Experimental Stimuli}

In this study, two types of emotion were produced by emotional video clips: sadness for negative emotion, happy for positive emotion. And these emotional video clips consisted of four categories which were classified by two axes of arousal (low to high arousal) and valence (negative to positive valence): positive-high arousal ( $\mathrm{PH}$ ), positive-low arousal (PL), negative-high arousal (NH), and negative-low arousal (NL). Based on previous studies, twenty-three video clips cut from part of a Korean movie were used for selection. ${ }^{23,29}$ At the first step of the selection, thirteen graduate students estimated the emotion, valence, arousal, and immersion state on each twenty-three video clips. To estimate emotion, participants had to choose one of the six basic emotion (happy, angry, sadness, fear, surprise, and disgust) to judge which emotions they experienced while watching the video clip. ${ }^{30}$ The level of valence, arousal, and immersion state were measured by Visual Analog Scale (VAS). ${ }^{31}$ The VAS consisted of 11 points on a $100 \mathrm{~mm}$ vertical line; valence scores ranged from 0 (very unpleasant) to 10 (very pleasant), arousal scores ranged from 0 (very relaxed) to 10 (very tense), and immersion scores ranged from 0 (never immersed) to 10 (very immersed). After that, twelve video clips were selected and were re-rated in the same way by sixty undergraduate students. Finally, four video clips were chosen for stimuli of the four categories and each video clip was matched to form a sequence of 3 minutes 30 seconds in length: PH (Forever the Moment), PL (The President's Barber), NH (Heart is ...), and NL (The Most Beautiful Goodbye). The score of each of the four video clips is presented in Table 1. During the experiment, the four video clips stimuli were presented on a 15 -inch CRT monitor with a $60-\mathrm{Hz}$ speaker from a distance of about $60 \mathrm{~cm}$.

\section{Manipulation Check}

The manipulation check was conducted to identify changes in the emotional states (emotion, valence, arousal,
Table I Mean ( \pm SD) for Valence, Arousal, and Immersion of Each Types of Stimuli

\begin{tabular}{|l|l|l|l|}
\hline Type of Stimuli & Valence & Arousal & Immersion \\
\hline $\mathrm{PH}$ & $7.97( \pm 0.89)$ & $7.30( \pm 1.56)$ & $7.80( \pm 1.27)$ \\
$\mathrm{PL}$ & $7.48( \pm 1.09)$ & $3.67( \pm 2.01)$ & $6.07( \pm 1.57)$ \\
$\mathrm{NH}$ & $2.93( \pm 1.17)$ & $7.27( \pm 1.39)$ & $7.73( \pm 1.08)$ \\
$\mathrm{NL}$ & $2.97( \pm 1.07)$ & $3.97( \pm 2.30)$ & $7.47( \pm 1.61)$ \\
\hline
\end{tabular}

Abbreviations: $\mathrm{PH}$, positive-high arousal condition; PL, positive-low arousal condition; NH, negative-high arousal condition; NL, negative-low arousal condition.

immersion) produced by the video clip stimuli. After viewing the video clips, participants were asked how they were feeling while watching the video clip. To estimate emotion, participants had to choose one of the six basic emotion: happy, angry, sadness, fear, surprise, and disgust. In addition, subjective valence, arousal level, and immersion induced by video stimuli were measured with the same kind of 11 points VAS which was used to choose experimental stimuli. Higher scores indicated a greater positive experience (happy), high level of arousal state, and immersion.

\section{Time Perception Task}

To measure the time perception objectively, a temporal bisection task was used. This task consisted of two phases: the practice phase, which teaches the participants to discriminate between short and long durations of presented cue, and the test phase, which measures to discriminate the presented cue with various durations. In the task, a crossfixation appeared for $500 \mathrm{~ms}$ on the monitor screen. Next, a blue circle with a $10 \mathrm{~cm}$ diameter was presented in the middle of the screen as a cue for judging the time. The cue of the practice phase was exposed for randomized durations of 400 or $1,600 \mathrm{~ms}$ and that of the test phase was exposed for randomized durations of 400, 600, 800, 1,000, $1,200,1,400$, or $1,600 \mathrm{~ms}$. After the cue disappeared, a left-arrow with the word "short" appeared on the left side of the screen and, simultaneously, a right-arrow with the word "long" appeared on the right side. The participants were asked to press the left-arrow if the blue circle was present for a short period of time or the right-arrow if the blue circle was present for a long period. Feedback was given following each trial in the practice phase to facilitate the participants' learning, but it was not given in the test phase. The 10 trials of the practice phase were followed by 35 trials in the test phase. The value of baseline of the time perception was measured by performing 10 trials practice phase before viewing the video clips, and 
35 trials of the test phase was repeated four times after viewing each video clip. Therefore, each participant performed 180 trials during the experiment. The temporal bisection task was presented using E-Prime 2.0 software (Psychology Software Tools, Inc).

\section{Procedures}

Each participant completed the experiment individually in a quiet laboratory, and the experimenters were blinded to the group difference of CD and ND. When participants arrived at the laboratory, they received information about the experimental procedure and completed the informed consent form. This was followed by a brief interview about their mental health history and the completion of the self-questionnaires including the PANAS and STAI-S. Subsequently, the time perception task was explained, the practice trials completed, and baseline time perception was measured at the practice phase. During the experiment phase, the participants viewed the video clips which were presented randomly, completed the time perception task, the manipulation check to confirm the level of induced emotional state, and were given a 5-minute break. The order of procedure from viewing the video clip to the break was repeated for each of the four types of video clip stimulus. The participants then completed a filler task, pressing the left-arrow whenever a circle appeared and the right-arrow whenever a square appeared on the monitor, for about 10 minutes to regain the initial mood state. Finally, they completed the PANAS and STAI-S questionnaires again. The entire experiment took approximately 1 hour and 15 minutes, and the participants were debriefed and provided with monetary compensation.

\section{Data Analysis}

Chi-square tests were used to analyze differences in characteristics of the CD and the ND groups. An independent sample $t$-test was used to analyze the group difference, and a paired sample $t$-test was used to analyze the differences between pre- and post-questionnaires for each group. The data from the temporal bisection task were compared between the two groups at baseline by calculating the Bisection Point (BP). The BP is a mid-point between the long and short length of presented durations. Based on the 0.5 $\mathrm{BP}$ value, a small value of BP means the internal clock speeds up, whereas a large value means the internal clock slows down. For example, a $0.3 \mathrm{BP}$ value indicates that participants judged time to pass quickly, whereas a $0.7 \mathrm{BP}$ value indicates that participants judged time to pass slowly.
In order to calculate the BP, we used the regression intercept and slope for each participant's responses at baseline. ${ }^{13}$ In addition, one-way analysis of variance (ANOVA) was used to examine differences in the patterns of time perception between two groups at baseline.

The value of time perception for each stimulus condition was calculated using the formula "each BP of participants in each condition - baseline BP." The zero value indicates the time perception was the same as the baseline. More distance from the zero-value indicated more changed in time perception compared to the baseline. A positive value indicated that a participant perceived time passing more slowly that at baseline, while a negative value indicated that a participant perceived time passing more quickly than at baseline. A 2 (group: CD or ND) betweensubject factor $\mathrm{x} 2$ (valence: positive or negative) $\mathrm{x} 2$ (arousal: high or low) within-subject factors mixed ANOVA was performed to identify differences in the pattern of time perception. Post-hoc tests were done to examine interactions in more detail.

The data of individuals who misunderstood the instructions, scored below $50 \%$ on accuracy in the practice phase of the temporal bisection task, or responded randomly in the test phase were excluded in data analysis. The data of three individuals of the CD group were excluded; one individual with comorbid learning disorder scored below $50 \%$ below in the practice phase and two individuals responded randomly in the test phase. All statistical data were analyzed with SPSS 17.0 for Windows.

\section{Results}

\section{Sample Characteristics}

There were no significant differences between the CD group and ND group in age $\left(\chi^{2}(1)=3.11, P=0.078\right)$, gender $\left(\chi^{2}(1)\right.$ $=0.83, P=0.362)$, marital status $\left(\chi^{2}(1)=2.54, P=0.111\right)$, employment status $\left(\chi^{2}(1)=0.02, P=0.888\right)$, and use of tobacco $\left(\chi^{2}(1)=0.07, P=0.791\right)$ or alcohol $\left(\chi^{2}(1)=0.51\right.$, $P=0.475)$.

\section{Characteristics of the Mood State}

The significant difference of mood state measured by selfreport questionnaires between the two groups were obtained. The CD group scored significantly higher on trait anxiety, state anxiety at pre-experiment, negative affective (NA) at pre-experiment, state anxiety at post-experiment and NA measured at post-experiment than the ND group. However, there were no differences between the two groups in positive 
affective (PA) state at pre-experiment and PA at postexperiment (Table 2). As a result of a paired sample $t$-test between pre- and post-score of self-report questionnaires, no significant differences were observed: state anxiety $(t(18)$ $=2.08, P=0.052)$, PA $(t(18)=1.17, P=0.257)$, and NA $(t(18)$ $=1.30, P=0.210)$ in the $\mathrm{CD}$ group. Additionally, there were no significant differences between pre- and post-experiment state anxiety $(t(21)=0.21, \quad P=0.836), \quad$ PA $\quad(t(21)=0.35$, $P=0.730)$, and NA $(t(21)=1.46, P=0.160)$ in the ND group.

\section{Manipulation Check}

The ND group had greater emotion recognition accuracy in the four conditions (PH, PL, NH, NL) than the CD group. With respect to the valence, arousal, and immersion, in the PH condition, the ND group perceived significantly greater positive valence than the CD group $(t(21)=$ $-2.74, P=0.012$ ), but there were no differences in arousal $(t(21)=-1.14, \quad P=0.267) \quad$ or $\quad$ immersion $\quad(t(21)=-1.89$, $P=0.073$ ) between the two groups. In the PL condition, the ND group perceived significantly greater positive valence $(t(21)=-9.88, P<0.001)$ and reported lower arousal $(t(21)=3.34, P=0.003)$ than the $\mathrm{CD}$ group. However, there was no difference in immersion $(t(21)=-1.73, P=0.098)$ between the two groups. Additionally, in the NH condition, the $\mathrm{CD}$ group perceived more significantly greater negative valence $(t(21)=-2.18, P=0.041)$ and reported lower arousal $(t(21)=-5.76, P<0.001)$ than the ND group. However, there was no difference in immersion $(t(21)=$ $-2.04, P=0.054)$ between the two groups. In the NL condition, the $\mathrm{CD}$ group perceived significantly greater negative valence than the ND group $(t(21)=-2.24, P=0.036)$. However, there were no differences in arousal $(t(21)=$ -0.08, $P=0.937)$ and immersion $(t(21)=1.47, P=0.156)$ between the two groups. All finding scores are presented in Table 3.

\section{Baseline and Each Stimulus Condition on Time Perception}

A one-way ANOVA was used to examine differences in time perception at baseline between the two groups. The results showed that there was a significant difference of time perception between the two groups $(F(1,39)=5.51$, $P=0.024$ ), indicating that the $\mathrm{CD}$ group perceived time passing more slowly than the ND group.

To compare the time perception on each valence and arousal condition between two groups, a 2 (group: CD or ND) x 2 (valence: positive or negative) x 2 (arousal: high or low) mixed ANOVA was used. The results revealed a significant interaction effect among group, valence, and arousal $\left(F(1,39)=6.02, P=0.019, \eta_{p}^{2}=0.13\right)$. To examine this interaction in more detail, two separate ANOVAs were conducted to identify the patterns of time perception according to the valence and arousal condition for each group (Figure 1). In order to control the increase of the type I error, the significance level was set by applying alpha correction $(0.05 / 6=0.008)$. As a result, in the CD group, there was a significant interaction effect between valence and arousal $\left(F(1,18)=28.26, P<0.001, \eta_{p}^{2}=0.61\right)$. In addition, there was no significant main effect of valence $\left(F(1,18)=5.18, \quad P=0.035, \quad \eta_{p}^{2}=0.22\right) \quad$ but there was a significant main effect in arousal $(F(1,18)=65.01$, $\left.P<0.001, \eta_{p}^{2}=0.78\right)$. The follow-up analyses revealed that the $\mathrm{CD}$ group perceived time passed more slowly in the NL condition compared to the PL condition $(F(1,72)=9.37$, $P=0.003$, and the NH condition $(F(1,72)=32.99, P<0.001)$. On the other hand, there were no differences of time

Table 2 Mean $( \pm S D)$ for Self-Questionnaires Between the CD Group and the ND Group

\begin{tabular}{|c|c|c|c|c|c|}
\hline & & $C D(N=19)$ & $N D(N=22)$ & $\mathbf{t}$ & $\boldsymbol{P}$ \\
\hline \multicolumn{2}{|l|}{ BDI-II } & $34.84( \pm 11.95)$ & $5.77( \pm 3.69)$ & 10.39 & $<0.001$ \\
\hline \multicolumn{2}{|l|}{ STAI-T } & $57.75( \pm \mid 5.31)$ & $36.95( \pm 6.97)$ & 5.54 & $<0.001$ \\
\hline \multirow[t]{3}{*}{ Pre-Questionnaires } & STAI-S & $57.00( \pm 14.91)$ & $35.59( \pm 7.53)$ & 6.14 & $<0.001$ \\
\hline & PANAS-PA & $27.47( \pm 8.95)$ & $30.05( \pm 4.75)$ & -0.97 & 0.345 \\
\hline & PANAS-NA & $31.00( \pm|2.6|)$ & $17.82( \pm 5.70)$ & 4.28 & $<0.001$ \\
\hline \multirow[t]{3}{*}{ Post-Questionnaires } & STAI-S & $49.74( \pm 15.13)$ & $35.18( \pm 6.99)$ & 4.06 & $<0.001$ \\
\hline & PANAS-PA & $24.84( \pm 9.17)$ & $29.59( \pm 6.11)$ & -1.67 & 0.112 \\
\hline & PANAS-NA & $27.68( \pm 8.5 \mathrm{I})$ & $16.09( \pm 5.82)$ & 4.17 & 0.001 \\
\hline
\end{tabular}

Abbreviations: CD, clinical-depressive group; ND, non-depressive group; BDI-II, Beck Depression Inventory-Second edition; STAI-S, State-Trait Anxiety Inventory-State Anxiety; STAI-T, State-Trait Anxiety Inventory-Trait Anxiety; PANAS-PA, The Positive Affect and Negative Affect Schedule-positive affect; PANAS-NA, The Positive Affect and Negative Affect Schedule-negative affect. 
Table 3 Accuracy of Emotional Perception and Mean $( \pm S D)$ for Valence, Arousal, and Immersion on Each Stimulus

\begin{tabular}{|l|l|l|l|l|l|}
\hline Group & Type of Stimuli & Accuracy & Valence & Arousal & Immersion \\
\hline $\mathrm{CD}$ & $\mathrm{PH}$ & $73.68 \%$ & $5.58( \pm 2.89)$ & $5.68( \pm 2.50)$ & $6.26( \pm 2.98)$ \\
& $\mathrm{PL}$ & $68.42 \%$ & $4.95( \pm 2.91)$ & $3.63( \pm 3.09)$ & $6.00( \pm 2.71)$ \\
& $\mathrm{NH}$ & $78.95 \%$ & $2.47( \pm 2.06)$ & $5.84( \pm 2.87)$ & $8.37( \pm 1.21)$ \\
& $\mathrm{NL}$ & $78.95 \%$ & $2.74( \pm 2.23)$ & $3.37( \pm 2.77)$ & $7.63( \pm 1.80)$ \\
\hline $\mathrm{ND}$ & $\mathrm{PH}$ & $90.91 \%$ & $7.36( \pm 1.50)$ & $6.41( \pm 2.52)$ & $7.68( \pm 1.81)$ \\
& $\mathrm{PL}$ & $94.45 \%$ & $7.41( \pm 1.94)$ & $3.05( \pm 2.63)$ & $7.09( \pm 1.51)$ \\
& $\mathrm{NH}$ & $90.91 \%$ & $3.55( \pm 1.57)$ & $7.00( \pm 1.95)$ & $9.50( \pm 2.11)$ \\
& $\mathrm{NL}$ & $94.45 \%$ & $4.23( \pm 1.34)$ & $3.55( \pm 2.04)$ & $7.09( \pm 1.95)$ \\
\hline
\end{tabular}

Abbreviations: $\mathrm{CD}$, clinical-depressive group; ND, non-depressive group; $\mathrm{PH}$, positive-high arousal condition; $\mathrm{PL}$, positive-low arousal condition; $\mathrm{NH}$, negative-high arousal condition; NL, negative-low arousal condition.

perception between the $\mathrm{PH}$ condition and the $\mathrm{NH}$ condition $(F(1,72)=0.90, P=0.346)$ and the PL condition $(F$ $(1,72)=3.00, P=0.088)$.

In the ND group, there were significant interaction effects between valence and arousal $(F(1,21)=13.63$, $\left.P=0.001, \quad \eta_{p}^{2}=0.39\right)$ and significant main effects of valence $\left(F(1,21)=110.46, P<0.001, \eta_{p}^{2}=0.84\right)$ and arousal $\left(F(1,21)=43.57, P<0.001, \eta_{p}^{2}=0.68\right)$. In follow-up analyses, it was found that the ND group perceived time passed more quickly in the PL condition than in the NL condition $(F(1,84)=10.16, P=0.002)$, the $\mathrm{PH}$ condition compared to the PL condition $(F(1,84)=24.73, P<0.001)$, and the NH condition compared to the NL condition ( $F$
$(1,84)=43.70, P<0.001)$. On the other hand, there were no differences in time perception between the $\mathrm{PH}$ condition and the NH condition $(F(1,84)=2.40, P=0.125)$. In addition, the results showed the main effects of valence ( $F$ $\left.(1,39)=53.30, \quad P<0.001, \quad \eta_{p}^{2}=0.58\right), \quad$ arousal $\quad(F(1,39)$ $\left.=79.82, P<0.001, \eta_{p}^{2}=0.67\right)$, and group $(F(1,39)=99.54$, $P<0.001, \eta_{p}^{2}=0.72$ ).

\section{Discussion}

The present study examined the effect of emotional state on depressive individuals' subjective time perception of neutral stimuli using a temporal bisection task. To do this, emotional states were induced using four types of video

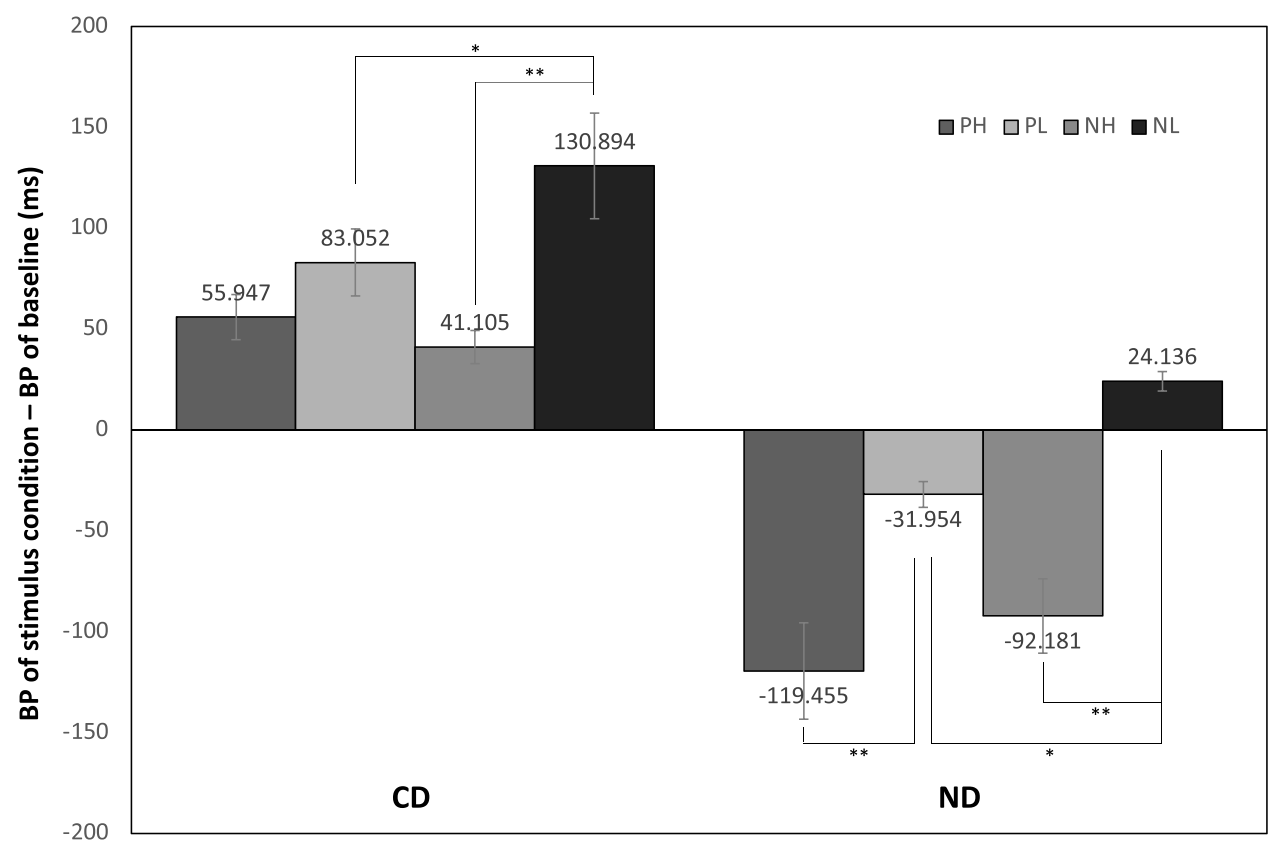

Figure I The change of time perception in valence and arousal conditions between the CD group and the ND group. Notes: $* P<0.01, * * P<0.001$.

Abbreviations: CD, clinical-depressive group; ND, non-depressive group; PH, positive-high arousal condition; PL, positive-low arousal condition; NH, negative-high arousal condition; NL, negative-low arousal condition. 
clips which were composed of arousal and valence: $\mathrm{PH}$, PL, NH, NL. Participants were asked to watch each type of video clip just before performing a temporal bisection task to induce emotional state, then they judged the flow of time of neutral stimuli.

The main results showed that, after mood induction, the depressive group perceived the time flows of neutral stimuli as slower than the non-depressive group in four stimuli condition as well as baseline. In accordance with previous studies, patients with depression generally perceived time passing more slowly in a depressive mood state. ${ }^{2,5}$ The other main results revealed that the nondepressive group perceived time passed more quickly in the positive stimuli with high-arousal condition than in the positive stimuli with low-arousal condition. In contrast, the depressive group did not show different patterns of time perception according to the level of arousal in positive valence condition. These results suggest that various aspects of attention resources should be considered in relation to time perception. Zakay and Block ${ }^{16}$ explained that when the external or additional information which attracts attention increases, the cognitive load increases, so there are not enough attention resources flowing into the switch. As a result of this distraction, time perception may be shorter. In addition, these results may be due to the different tendency of response on emotional information between the two groups, as it is known that individuals with depression mood show higher insensitivity to positive emotions than non-depressive individual. ${ }^{32}$ It means that the non-depressive group was more attracted to positive stimuli than the depressive group. As they showed a high level of immersion to positive stimuli, the non-depressive group was likely to perceive the time quickly on temporal bisection task due to the prolongation of emotional assimilation to positive stimuli.

On the manipulation check of stimuli, the depressive group was less accurate in emotion recognition than the non-depressive group. Specifically, the depressive group reported non-response, did not feel any emotion, or scored lower on the positive stimuli than the non-depressive group. The depressive group also perceived more negativity in the negative stimuli than the non-depressive group. These results coincide with previous results. ${ }^{7,33}$ Thus, these findings suggest that imbalanced emotional experiences could influence the perception of time in individuals with depression, and this may be a result of differences in the emotional experiences in the two groups.
The previous studies suggested that the perception of time is a dual mechanism (attention and emotion) based on the arousal. In accordance with these theories, the current results showed that negative stimuli slowed the perception of time passing compared to positive stimuli in lowarousal conditions for both groups and, thus, these findings support the dual mechanism in low-arousal condition explanation. ${ }^{12,19}$ The dual mechanism theory also suggests that in the high-arousal condition, negative stimuli lead to an increase of the speed of the internal clock, while positive stimuli lead to a decrease in the speed. The response to negative stimuli is more related to survival than the response to positive stimuli, and increased threat level triggers an increase in the speed of the internal clock. However, the current study found that there was no significant difference according to the valence in the higharousal condition. This result is consistent with a previous study, which found the valence effect did not influence the perception of time in the high-arousal condition. ${ }^{12}$ It is possible to assume that humans can automatically show attention avoidance to negative stimuli for emotional regulation at high arousal level, so it is estimated that the attentional resources to perceive time become diminished, resulting in slower perception of time. ${ }^{20}$ Considering that the main factor in the operation of the internal clock is the arousal response and the role of valence is a modulating variable, our result suggests that the mechanism of time perception depends on the strength of arousal in the emotional context. ${ }^{13,14}$

These findings have several implications. First, the current study investigated that valence and arousal factors could change the time perception in individuals with depression. Therefore, modifying the distorted time perception considering the valence and arousal factors could be useful in alleviating the slowed perception of time and depression induced by this perception. Second, this study provides evidence that understanding the process of the perception of time requires consideration of the influence of valence and arousals on the model of the internal clock.

Despite its contributions, the present study has some limitations. First, the generalization of these results may be difficult due to the use of medication and comorbid disorders in the CD group. Therefore, further research should be conducted with a large sample to control the extraneous variables. Second, the current study did not measure the other depressive symptoms such as cognitive and psychomotor functions, loss of energy, and ruminations. ${ }^{34,35}$ Because these symptoms could influence the perception 
of time, they should be considered in future studies. Third, this study did not measure whether the mood induction lasted the same amount of time for the depressive group and non-depressive group. Future research will need to check these aspects to clarify the effect of induced emotional states on time perception.

\section{Conclusion}

The present study demonstrated that the perception of time was affected by valence and arousal factors in individuals with depression, as well as individuals without depression. These findings have implications for the treatment of depression. It is important to consider valence as well as arousal in the treatment of depression to remedy the distorted perception of time and change the emotional experiences in individuals with depression. In addition, the results suggest an extension of the theoretical internal clock model through the inclusion of the effects of valence and arousal.

\section{Acknowledgments}

The authors thank the patients for participation.

\section{Disclosure}

The authors report no conflicts of interest in this work.

\section{References}

1. Droit-Volet S, Meck WH. How emotions colour our perception of time. Trends Cogn Sci. 2007;11(12):504-513. doi:10.1016/j. tics.2007.09.008

2. Droit-Volet S, Gil S. The time-emotion paradox. Philos Trans $R$ Soc Lond B Biol Sci. 2009;364(1525):1943-1953. doi:10.1098/ rstb.2009.0013

3. Blewett AE. Abnormal subjective time experience in depression. $\mathrm{Br}$ J Psychiatry. 1992;161:195-200. doi:10.1192/bjp.161.2.195

4. Kitamura T, Kumar R. Time passes slowly for patients with depressive state. Acta Psychiat Scand. 1982;4:127-140.

5. Bschor $T$, Ising $M$, Bauer $M$, et al. Time experience and time judgment in major depression, mania and healthy subjects. A controlled study of 93 subjects. Acta Psychiat Scand. 2004;109:222-229. doi:10.1046/j.0001-690X.2003.00244.x

6. Hoffer A, Osmond H. The relationship between mood and time perception. Psychiatr Q Suppl. 1962;36:87-92.

7. Bylsma LM, Morris BH, Rottenberg J. A meta-analysis of emotional reactivity in major depressive disorder. Clin Psychol Rev. 2008;28:676-691. doi:10.1016/j.cpr.2007.10.001

8. Treisman M. Temporal discrimination and the indifference interval: implications for a model of the "Internal clock". Psychol Monogr. 1963;77:1-31. doi:10.1037/h0093864

9. Church RM. Properties of the internal clock. Ann NY Acad Sci. 1984;423:566-582. doi:10.1111/j.1749-6632.1984.tb23459.x

10. Wilkie DM. Stimulus intensity affects pigeons' timing behavior: implications for an internal clock model. Anim Learn Behav. 1987;15:35-39. doi:10.3758/BF03204901
11. Burle B, Casini L. Dissociation between activation and attention effects in time estimation: implications for internal clock models. $J$ Exp Psychol-Hum Percept Perform. 2001;27:195-205. doi:10.1037/0096-1523.27.1.195

12. Gil S, Droit-Volet S. Emotional time distortions: the fundamental role of arousal. Cogn Emot. 2012;26(5):847-862. doi:10.1080/ 02699931.2011.625401

13. Smith SD, McIver TA, Di Nella MS, Crease ML. The effects of valence and arousal on the emotional modulation of time perception: evidence for multiple stages of processing. Emotion. 2011;11:1305-1313. doi:10.1037/a0026145

14. Mella N, Conty L, Pouthas V. The role of physiological arousal in time perception: psychophysiological evidence from an emotion regulation paradigm. Brain Cogn. 2011;75:182-187. doi:10.1016/j. bandc.2010.11.012

15. Msetfi RM, Murphy RA, Kornbrot DE. The effect of mild depression on time discrimination. $Q J$ Exp Psychol. 2012;65:632-645. doi:10.1080/17470218.2011.608908

16. Zakay D, Block RA. Temporal cognition. Curr Dir Psychol Sci. 1997;6(1):12-16. doi:10.1111/1467-8721.ep11512604

17. Bech P. Depression: influence on time estimation and time experiments. Acta Psychiat Scand. 1975;51:42-50. doi:10.1111/ j.1600-0447.1975.tb00211.x

18. Russell JA. A circumplex model of affect. J Pers Soc Psychol. 1980;39:1161-1178. doi:10.1037/h0077714

19. Angrilli A, Cherubini P, Pavese A, Manfredini S. The influence of affective factors on time perception. Percept Psychophys. 1997;59:972-982. doi:10.3758/BF03205512

20. Dolcos F, Bogdan PC, O'Brien M, et al. The impact of focused attention on emotional evaluation: a eye-tracking investigation. Emotion. 2020. doi:10.1037/emo0000895

21. Gil S, Droit-Volet S. "Time flies in the presence of angry faces" ... depending on the temporal task used! Acta Psychol (Amst). 2011;136 (3):354-362. doi:10.1016/j.actpsy.2010.12.010

22. Thönes S, Oberfeld D. Time perception in depression: a meta-analysis. J Affect Disord. 2015;175:359-372. doi:10.1016/j. jad.2014.12.057

23. Lee SJ, Choi ND. Selecting film excerpts optimal for eliciting basic emotions and the systematic understanding of the evoked emotional experiences. Kor J Broadcast Telecomm Stud. 2009;23:205-246.

24. Westermann R, Spies K, Stahl G, Hesse FW. Relative effectiveness and validity of mood induction procedures: a meta-analysis. Eur $J$ Soc Psychol. 1996;26:557-580. doi:10.1002/(SICI)1099-0992(199607)26:4<557::AID-EJSP769>3.0.CO;2-4

25. Schaefer A, Nils F, Sanchez X, Philippot P. Assessing the effectiveness of a large database of emotion-eliciting films: a new tool for emotion researchers. Cogn Emot. 2010;24:1153-1172. doi:10.1080/ 02699930903274322

26. Beck AT, Steer RA, Brown GK. Manual for the Beck Depression Inventory-II. Texas: Psychological Corporation; 1996.

27. Spielberger CD, Gorsuch RL, Lushene RE. Manual for the State-Trait Anxiety Inventory. California: Consulting Psychologist Press; 1983.

28. Watson D, Clark LA, Tellegen A. Development and validation of brief measures of positive and negative affect: the PANAS scales. $J$ Pers Soc Psychol. 1988;54:1063-1070. doi:10.1037/00223514.54.6.1063

29. Jang EH, Suk JA, Eom JS, Sohn JH. Development of protocols for inducing emotions using audio-visual film clips. Kor J Exp Psychol. 2005; 17:69-84.

30. Ekman P. An argument for basic emotions. Cogn Emot. 1992;6:169-200. doi:10.1080/02699939208411068

31. Huskisson EC. Visual analogue scales. In: Melzack R, editor. Pain Measurement and Assessment. New York: Raven Press; 1983:33-37.

32. Weightman MJ, Air TM, Baune BT. A review of the role of social cognition in major depressive disorder. Front Psychiatr. 2014;5:179. doi:10.3389/fpsyt.2014.00179 
33. Watson D, Clark LA, Carey G. Positive and negative affectivity and their relation to anxiety and depressive disorders. J Abnorm Psychol. 1988;97(3):346-353. doi:10.1037/0021-843X.97.3.346

34. American Psychiatric Association. Diagnostic and Statistical Manual of Mental Disorders. Fourth ed. Washington, DC: American Psychiatric Association; 2000.
35. Nolen-Hoeksema S. Responses to depression and their effects on the duration of depressive episodes. $J$ Abnorm Psychol. 1991;100:569-582. doi:10.1037/0021-843X.100.4.569

\section{Publish your work in this journal}

Psychology Research and Behavior Management is an international, peer-reviewed, open access journal focusing on the science of psychology and its application in behavior management to develop improved outcomes in the clinical, educational, sports and business arenas. Specific topics covered in the journal include: Neuroscience, memory and decision making; Behavior modification and management; Clinical applications; Business and sports performance management; Social and developmental studies; Animal studies. The manuscript management system is completely online and includes a very quick and fair peer-review system, which is all easy to use. Visit http://www. dovepress.com/testimonials.php to read real quotes from published authors. 